\title{
Eficacia de la adición de clopidogrel a ácido acetilsalicílico en el infarto agudo de miocardio con elevación del segmento ST
}

En pacientes con infarto agudo de miocardio el añadir clopidogrel (75 mg)

a la antiagregación con ácido acetilsalicílico reduce un $0,9 \%$ el riesgo de mortalidad o reinfarto o ictus a los 28 días, sin un incremento de los efectos adversos.

In patients with acute myocardial infarction adding clopidogrel (75 $\mathrm{mg}$ ) to aspirin reduces $0.9 \%$ the combined risk of mortality, reinfarction or stroke within the first 28 days, without any significant adverse effect.

Artículo: Chen ZM, Jiang LX, Chen YP, Xie JX, Pan HC, Peto R, et al, COMMIT (ClOpidogrel and Metroprolol in Myocardial Infarction Trial) collaborative group. Addition of clopidogrel to aspirin in 45,852 patients with acute myocardial infarction: randomised placebo-controlled trial. Lancet. 2005;366:1607-21.

Antecedentes: las plaquetas desempeñan un papel importante en la formación del trombo tras la ruptura de la placa arteriosclerótica que inicia habitualmente el síndrome coronario agudo. Además, las plaquetas se activan precozmente tras el tratamiento trombolítico, favoreciendo la actividad antifibrinolítica. La doble antiagregación plaquetaria mediante el empleo de ácido acetilsalicílico, que bloquea la enzima ciclooxigenasa, y clopidogrel, que bloquea la unión de ADP a los receptores específicos plaquetarios $\left(\mathrm{P} 2 \mathrm{Y}_{12}\right)$, ha sido evaluada en un ensayo clínico de 12.562 enfermos con síndrome coronario agudo sin elevación del segmento $\mathrm{ST}^{1}$, con un alto porcentaje de factores de riesgo. La adición de clopidogrel a ácido acetilsalicílico se asoció a una reducción de la incidencia de infarto, del $6,7 \%$ al $5,2 \%$, y a un incremento del riesgo de sangrado grave no fatal del $2,7 \%$ al $3,7 \%$ El ensayo CLARITY2 incluyó 3.491 enfermos con infarto agudo de miocardio (IAM) con elevación del segmento ST y menores de 75 años. La adición de clopidogrel a ácido acetilsalicílico se asoció a una reducción de la incidencia de reinfarto del 3,6 \% al 2,5\%, sin modificación de la mortalidad, que fue 2,2\% en el grupo ácido acetilsalicílico frente a 2,6\% en el grupo clopidogrel más ácido acetilsalicílico. No hubo aumento del riesgo de sangrado.

Objetivo: estimar en los pacientes con IAM el efecto del tratamiento que asocia clopidogrel y ácido acetilsalicílico frente a ácido acetilsalicílico sobre un desenlace combinado de muerte de cualquier causa, reinfarto o ictus.

Tabla 1: Diseño factorial

\begin{tabular}{|l|c|c|c|}
\hline \multirow{2}{*}{ Metoprolol } & & Clopidogrel & Grupos resultantes \\
& Clopidogrel + metoprolol & Placebo clopidogrel + metoprolol & $\begin{array}{c}\text { Metoprolol } \\
\mathrm{n}=22.929\end{array}$ \\
\cline { 2 - 5 } & Clopidogrel + placebo metoprolol & Placebo clopidogrel + placebo metoprolol & $\begin{array}{c}\text { Placebo metoprolol } \\
\mathrm{n}=22.923\end{array}$ \\
\hline Grupos resultantes & $\begin{array}{c}\text { Clopidogrel } \\
\mathrm{n}=22.961\end{array}$ & $\begin{array}{c}\text { Placebo clopidogrel } \\
\mathrm{n}=22.891\end{array}$ & $\mathrm{n}=45.852$ \\
\hline
\end{tabular}

El diseño factorial $2 \times 2$ permite comparar en los mismos enfermos 2 tipos de intervenciones.

*Esta sección está financiada con la ayuda ISCIII-FIS 02/8115. 
Tabla 2: Características generales

\begin{tabular}{|l|c|c|}
\hline & $\begin{array}{c}\text { Grupo clopidogrel y ácido acetilsalicílico } \\
(\mathrm{n}=22.961)\end{array}$ & $\begin{array}{c}\text { Grupo ácido acetilsalicílico } \\
(\mathrm{n}=22.891)\end{array}$ \\
\hline Edad, años, media & 61 & 61 \\
$\geq 70$ años & $26 \%$ & $26 \%$ \\
Género, mujeres, \% & 28 & 28 \\
Tiempo desde inicio síntomas, horas, media* & 10,3 & 10,3 \\
Tensión arterial sistólica, mmHg, media & 128 & 128 \\
Frecuencia cardíaca, Ipm, media & 82 & 82 \\
ECG inicial, \% & & 87 \\
Elevación ST & 87 & 6 \\
Bloqueo de rama izquierda & 7 & 7 \\
Descenso del segmento ST & 7 & 24 \\
Killip II o III, \% & 25 & 8 \\
Patología previa, \% & & 43 \\
Infarto agudo de miocardio & 9 & 18 \\
Hipertensión arterial & 43 & 7 \\
Tratamiento médico previo, \% & & 50 \\
Ácido acetilsalicílico & 18 & \\
Bloqueadores beta & 6 & 94 \\
Fibrinólisis antes de la aleatorización & 50 & 75 \\
Tratamiento durante hospitalización, \% & & 68 \\
Nitratos & 94 & 54 \\
Anticoagulantes & 74 & 22 \\
Inhibidores de la enzima conversora de angiotensina & 68 & 12 \\
Fibrinolítico & 54 & 10 \\
Antiarrítmicos & 22 & 3 \\
Antagonistas del calcio & 12 & \\
Otros antiagregantes & 10 & 3 \\
Procedimientos percutáneos electivos, \% & 3 & \\
\hline
\end{tabular}

*No se especifica en el texto si el tiempo fue desde el comienzo de los síntomas hasta la asignación aleatoria o hasta recibir el tratamiento. "Principalmente heparina.

₹Principalmente uroquinasa.

Diseño: ensayo clínico aleatorizado. Se planteó un diseño factorial $2 \times 2$ (tabla 1) para valorar de modo independiente la seguridad y la eficacia del metoprolol frente a placebo y la de la asociación de clopidogrel con ácido acetilsalicílico frente a ácido acetilsalicílico en estos pacientes.
Lugar: mil doscientos cincuenta hospitales en China.

Período de estudio: de agosto de 1999 a febrero de 2005 .

Pacientes (tabla 2 y figura 1 ): Criterios de inclusión: pacientes con sospecha de IAM de menos de 24 horas de evolución

Figura 1. Diagrama de inclusión

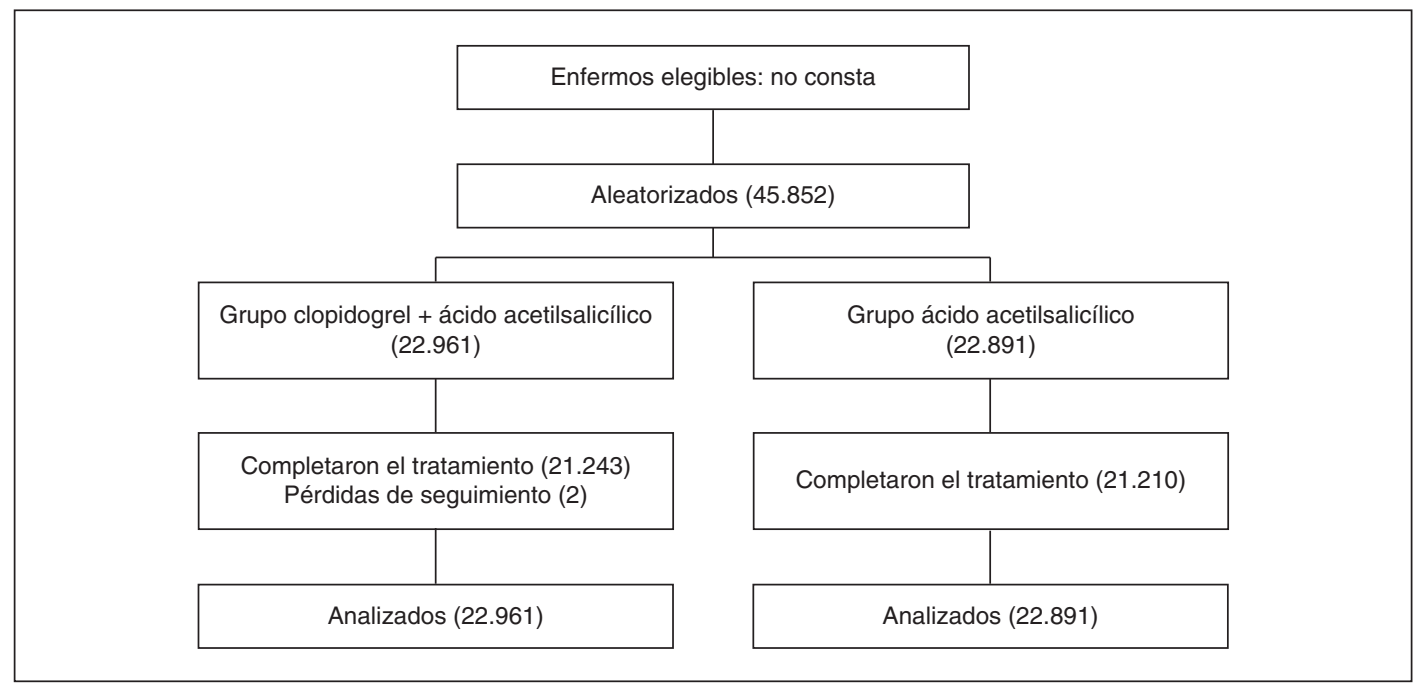


Tabla 3: Desenlace combinado de muerte de cualquier causa, reinfarto o ictus durante el período de estudio

\begin{tabular}{|lc|}
\hline Grupo clopidogrel y ácido & \\
$\quad$ acetilsalicílico & $2.121 / 22.961(9,2 \%)$ \\
Grupo ácido acetilsalicílico & $2.310 / 22.891(10,1 \%)$ \\
RR (IC 95\%) & $0,92(0,87$ a 0,97$)$ \\
RRR (IC 95\%) & $8,5 \%(3,1 \%$ a $13,4 \%)$ \\
RAR (IC 95\%) & $0,9 \%(0,3 \%$ a $1,4 \%)$ \\
NNB (IC 95\%) & $118(74$ a 314$)$ \\
\hline
\end{tabular}

con elevación del segmento ST, bloqueo de rama izquierda o descenso del segmento ST en el electrocardiograma inicial, en los que el médico responsable no considerara que había indicación o contraindicación para el empleo de alguno de los fármacos del estudio.

Criterios de exclusión: 1. pacientes en los que se decidió tratamiento del IAM mediante intervencionismo coronario primario. 2. poca probabilidad de beneficio potencial por una historia clínica no convincente de IAM o por una enfermedad concomitante de mal pronóstico vital. 3. alto riesgo de efectos adversos de las medicaciones del estudio (alergia previa a ácido acetilsalicílico o sangrado activo o historia de alteración previa de la hemostasia o hipotensión persistente o bradicardia persistente o shock cardiogénico o bloqueo de alto grado). 4. ausencia de consentimiento informado.

\section{Intervenciones que se comparan:} Grupo clopidogrel: ácido acetilsalicílico $162 \mathrm{mg} /$ día + clopidogrel 75 mg/día ( $\sin$ dosis de carga).

Grupo placebo: ácido acetilsalicílico $62 \mathrm{mg} /$ día + placebo.

La duración del tratamiento fue de 4 semanas o hasta el alta hospitalaria o la muerte si ocurrían antes. El resto del tratamiento fue a criterio de los médicos responsables, excepto el tratamiento con metoprolol, que también fue aleatorizado. En los enfermos que recibieron fibrinólisis el tratamiento se comenzó generalmente antes de ésta.
Tabla 4: Desenlace de muerte de cualquier causa durante el período de estudio

\begin{tabular}{|lc|}
\hline Grupo clopidogrel y ácido & \\
$\quad$ acetilsalicílico & $1.726 / 22.961(7,5 \%)$ \\
Grupo ácido acetilsalicílico & $1.845 / 22.891(8,1 \%)$ \\
RR (IC 95\%) & $0,93(0,88$ a 0,99$)$ \\
RRR (IC 95\%) & $6,7 \%(0,6 \%$ a $12,8 \%)$ \\
RAR (IC 95\%) & $0,5 \%(0,1 \%$ a $1,0 \%)$ \\
NNB (IC 95\%) & $184(97$ a 1.914$)$ \\
\hline
\end{tabular}

La incidencia de reinfarto fue 2,4\% en el grupo ácido acetilsalicílico y $2,1 \%$ en el grupo clopidogrel y ácido acetilsalicílico $(\mathrm{p}=0,02)$.

La incidencia de ictus fue $1,1 \%$ en el grupo ácido acetilsalicílico y $0,9 \%$ en el grupo clopidogrel y ácido acetilsalicílico $(\mathrm{p}=0,11)$.

Asignación aleatoria: sí. Oculta.

Enmascaramiento: sí.

Desenlaces principales: se plantearon dos objetivos primarios: 1 . desenlace combinado de muerte por cualquier causa, reinfarto o ictus; 2 . muerte por cualquier causa durante los primeros 28 días o hasta el alta hospitalaria si se producía antes.

Las definiciones de reinfarto y de ictus están adecuadamente referidas en el texto.

\section{Análisis por intención de tratar: sí.}

Resultados principales (tablas 3, 4 y 5): la duración media del tratamiento fue de 14,9 días en los supervivientes. Los resultados obtenidos son aparentemente independientes de las características de los pacientes al ingreso, por ejemplo, edad $\geq 70$ años, y de otros tratamientos recibidos, por ejemplo fibrinolíticos.

Información sobre costes: no consta.

Financiación del estudio: el estudio fue financiado por Sanofi-Aventis, Bristol-Myers, Astra-Zeneca, UK Medical Research Council, British Heart Foundation y Cancer Research UK.

Conflicto de intereses: están adecuadamente descritos en el texto.

Tabla 5: Complicaciones durante el estudio

\begin{tabular}{|l|c|c|}
\hline & $\begin{array}{c}\text { Grupo clopidogrel y ácido acetilsalicílico } \\
(\mathrm{n}=22.961)\end{array}$ & $\begin{array}{c}\text { Grupo ácido acetilsalicílico } \\
(\mathrm{n}=22.891)\end{array}$ \\
\hline Complicación hemorrágica fatal, \% & 0,32 & 0,32 \\
Complicación hemorrágica no fatal, \% & 0,27 & 0,22 \\
\hline
\end{tabular}


Conclusiones de los autores: en pacientes con IAM, añadir 75 mg/día, sin dosis de carga, de clopidogrel al tratamiento con ácido acetilsalicílico reduce la mortalidad y la incidencia de eventos vasculares mayores, sin aumentar la incidencia de complicaciones.

Conclusiones de los revisores: los resultados del estudio COMMIT son congruentes con los de ensayos clínicos previos 1-3 en los que se explora el uso de la doble antiagregación en pacientes con síndrome coronario agudo con o sin elevación del segmento ST. El estudio COMMIT demuestra una reducción absoluta de la mortalidad de cualquier origen, reinfarto o ictus del $0,9 \%$ y de muerte a los 28 días del $0,5 \%$. Es decir, es preciso tratar a 184 pacientes para salvar una vida, mostrando probablemente el empleo de clopidogrel una adecuada relación coste/beneficio. Sin embargo, los resultados del presente estudio deben ser tomados con cautela a la hora de generalizarlos a otros medios. Este estudio fue realizado exclusivamente en China y la pauta de tratamiento empleada difiere significativamente de la práctica habitual de las unidades de nuestro entorno. Así, por ejemplo, sólo un 50\% de los pacientes estudiados recibieron tratamiento fibrinolítico, que además fue realizado con uroquinasa. Se realizó un cateterismo únicamente en menos de un $5 \%$ de los pacientes estudiados.

Este ensayo clínico presenta aspectos que son relevantes en la práctica clínica. En primer lugar se han incluido enfermos con edad $\geq 70$ años (26\%). En este subgrupo de pacientes con mayor edad la eficacia es similar a la observada en los grupos con edad inferior, y no se ha observado un incremento del riesgo de sangrado, por lo que este subgrupo de población no debe ser excluida a priori del tratamiento con doble antiagregación.

En el presente estudio no se empleó la dosis de carga de 300 mg de clopidogrel, cuya justificación es inducir una inhibición más rápida de los receptores plaquetarios de ADP. Los autores prescindieron de la dosis de carga con el fin de disminuir el riesgo potencial de sangrado inducido por al dosis de carga, por ejemplo, enfermos ancianos que reciben tratamiento fibrinolítico. Los hallazgos del ensayo muestran que la reducción de la incidencia del desenlace combinado en el grupo con doble antiagregación es ya apreciable desde el primer día. Por tanto, el beneficio/riesgo de la dosis de carga de clopidogrel es dudoso y posiblemente deba ser evaluado en el futuro.

\section{Bibliografía}

1. Yusuf S, Zhao F, Mehta SR, Chrolavicius S, Tognoni G, Fox KK. Clopidogrel in Unstable Angina to Prevent Recurrent Events Trial Investigators. Effects of clopidogrel in addition to aspirin in patients with acute coronary syndromes without STsegment elevation. N Engl J Med. 2001;345:494-502.

2. Sabatine MS, Cannon CP, Gibson CM, López-Sendón JL, Montalescot G, Theroux P, et al. Addition of clopidogrel to aspirin and fibrinolytic therapy for myocardial infarction with ST-segment elevation. N Engl J Med. 2005;352:1179-89.
3. Blancas Gómez-Casero R, Nevado Losada E, López Matamala B, Martínez Díaz C, Serrano Castañeda J, González Manzanares JL, et al. Empleo conjunto de tirofibán y clopidogrel en el síndrome coronario agudo sin elevación del segmento ST de alto riesgo. Med Intensiva. 2005;29:373-8. 